\title{
Varietätengebrauch und Varietätenkontakt in Südtirol und Ostbelgien
}

\author{
Claudia Maria Riehl (Köln)
}

\begin{abstract}
Dialect often plays an important role in minority communities where it functions as a marker of ethnic identity. In this case it also becomes an issue for speakers of the majority group who intend to acquire the minority language. The situation, however, differs from region to region and within different minority groups.

The article discusses the linguistic setting and variety use of two German-speaking minorities, South Tyrol and East Belgium. The main focus is on the dimensions of dialect use in different domains, its linguistic influence on the standard variety, and its role for identity building. It will be pointed out that South Tyrolians almost exclusively identify with their regional dialect, whereas East Belgians also make use of language mixing.

In its conclusion the article emphasizes the importance of dialectal and regional varieties for L2-learners: Learners should not only acquire a passive knowledge of the respective minority dialects, but also come to appreciate its symbolic value in the respective communities.
\end{abstract}

\section{$1 \quad$ Einleitung}

In vielen Sprachminderheitsgebieten wird neben der Standardvarietät der Minderheitensprache ein Dialekt verwendet, der meistens auch eine zentrale Bedeutung für den Ausdruck der Identität der Minderheiten hat. In diesen Fällen wird der Dialekt dann auch wichtig für die anderssprachige Bevölkerung dieser Regionen, die die Minderheitensprache als Zweitsprache erwerben will oder muss. Zwei dieser Regionen, in denen eine dialektale Varietät des Deutschen als Minderheitensprache eine zentrale Rolle spielt, sind Südtirol und Ostbelgien. Beide unterscheiden sich jedoch in der Intensität der Dialektverwendung, des Varietätenkontakts und in der Rolle des Dialekts für die Konstitution einer Minderheitenidentität.

Im Folgenden soll die sprachliche Situation und der Varietätengebrauch in diesen Regionen vorgestellt und davon ausgehend die Einstellung der Sprecher gegenüber dem Dialekt und anderen Varietäten dargelegt werden. Danach werden die Einflüsse des Kontakts der dialektalen Varietäten auf die Standardsprache beschrieben und damit die Problematik einer regionalen Norm diskutiert. Schließlich wird darauf hingewiesen, welche Konsequenzen sich daraus für den Erwerb des Deutschen als Zweitsprache in diesen Gebieten ergeben.

\section{$2 \quad$ Zur sprachlichen Situation in Südtirol und Ostbelgien}

\subsection{Südtirol}

Aufgrund des Autonomiestatuts verfügt die deutsche Minderheit in Südtirol über zahlreiche Rechte, die die kulturelle und sprachliche Eigenständigkeit sichern. So werden etwa alle öffentlichen Zuschüsse für Kultur und soziale Fürsorge im Verhältnis zur zahlenmäßigen 
Stärke der Sprachgruppen zugeteilt (sog. Proporzregelung); Deutsch ist dem Italienischen gleichgestellt und Deutschsprachige haben das Recht, mit allen Stellen jederzeit Deutsch zu sprechen (cf. Egger/Heller 1997, Eichinger 1996: 214-223). Aus diesem Grund ist die deutsche Sprache nicht nur in den öffentlichen zweisprachigen Aufschriften, sondern auch in den Medien stark vertreten (etwa durch eigene Tageszeitungen, Magazine, Radiosender und deutschsprachige Fernsehsendungen). Das Deutsche hat auch im Bereich der Schule seine feste Position. In den Schulen der deutschen Sprachgruppe ist Deutsch alleinige Unterrichtssprache, die Zweitsprache Italienisch wird mit einem Anteil von ca. 6 Wochenstunden ab der zweiten Klasse Primarschule gelehrt. Umgekehrt gibt es für die italienische Sprachgruppe die gleichen Schultypen mit jeweils Italienisch als Unterrichtssprache und Deutsch als Zweitsprache ab der zweiten Klasse Primarschule. Der Unterricht wird dabei von Lehrern erteilt, für die die jeweilige Sprache Muttersprache ist (cf. Riehl 2000: 241-242).

In Südtirol ist die regionale Varietät des Deutschen, nämlich der Südtiroler Dialekt, fast ausschließliches Kommunikationsmedium im mündlichen Sprachgebrauch der deutschen Sprachgruppe, und zwar in allen Generationen. Daneben wird Standarddeutsch in formellen Situationen und im Umgang mit Touristen oder den italienischsprachigen Mitbürgern verwendet. Allerdings gebraucht man in letzterem Fall viel häufiger die italienische Sprache. (cf. Egger/Heller 1997: 1350). Deutsch wird auch im schriftlichen Medium in offiziellen und inoffiziellen Schriftwechseln, Verwaltung etc. gleichberechtigt neben Italienisch verwendet.

\subsection{Ostbelgien}

Anders ist die Situation in Ostbelgien, und zwar in der sog. Deutschsprachigen Gemeinschaft. ${ }^{1}$ Die Gemeinschaft besitzt eine eigene Verwaltung innerhalb des belgischen Föderalstaats und ist einsprachig deutsch, d. h. in Gesetzgebung, Verwaltung und öffentlichen Aufschriften (wichtige Verkehrshinweise und Bekanntmachungen ausgenommen). Die Gemeinschaft verfügt darüber hinaus über eine eigene Tageszeitung und einen Radiosender sowie ein deutschsprachiges Magazin im staatlichen Fernsehsender (cf. Hinderdael/Nelde 1996).

Auch hier hat die deutsche Sprache ihre Position in der Schule: Der Unterricht ist einsprachig deutsch, die Zweitsprache wird ab der 1. bis 3. Klasse Primarschule mit 6 Wochenstunden gelehrt; in den letzten drei Klassen der Oberschule werden auch einige weitere Fächer, v. a. naturwissenschaftliche Fächer und Mathematik, auf Französisch unterrichtet. Hier steht es den Schulen bis zu einem gewissen Grad frei, inwiefern sie den Unterricht zweisprachig gestalten wollen: Es können sogar bis zu 2/3 des Unterrichts in französischer Sprache abgehalten werden (Riehl 2001: 36-38). In Ostbelgien wird - wie in Südtirol - innerhalb der deutschsprachigen Gruppe eine Varietät des Deutschen gesprochen: im südlichen Teil der Gemeinschaft und bei der älteren Generation noch häufig der jeweilige Dialekt, das sog. Platt (beruhend auf mosel- und rheinfränkischen Dialekten), ${ }^{2}$ im nördlichen Teil und bei der jüngeren Generation eine regionale Umgangssprache, die sich an die rheinische Umgangssprache anlehnt und Übernahmen aus der Kontaktsprache zeigt (cf. Riehl 2001: 3940). Die schriftliche Kommunikation erfolgt aufgrund der offiziellen Einsprachigkeit des Gebietes ebenfalls auf Deutsch. Allerdings sind aufgrund der geringen Größe der Region und wirtschaftlichen Zugehörigkeit zur Wallonie überregionale Schriftwechsel auf Französisch zu führen. Mit frankophonen Mitbürgern kommuniziert man fast ausnahmslos in französischer

\footnotetext{
1 Weitere Gebiete wie der Kanton Malmedy und Regionen des sog. 'Altbelgiens', in denen für die deutsche Sprachgemeinschaft allenfalls "Erleichterungen" bestehen, werden hier ausgeklammert. Zur komplexen Situation im deutschsprachigen Belgien cf. Hinderdael/Nelde (1996).

2 Zur Einteilung der Dialekte auf ostbelgischem Gebiet cf. etwa Kern (1997).
} 
Varietätengebrauch und Varietätenkontakt in Südtirol und Ostbelgien

Sprache. Außerdem werden in fast allen Berufen Kenntnisse in beiden Sprachen vorausgesetzt.

\section{Varietätengebrauch}

Auch in der Minderheitensituation zeigt sich ein ähnliches Bild wie in der übrigen Dialektlandschaft des Deutschen, nämlich ein sehr starkes Nord-Süd-Gefälle: In Südtirol deckt der Dialekt noch nahezu alle Domänen gesprochener Sprache ab, in Ostbelgien tut dies in zunehmendem Maße eine regionale Standardsprache. Allerdings bestehen hier große Unterschiede zwischen der Situation im Kanton Eupen und im Kanton St. Vith. Letzterer ist aufgrund seiner stärkeren geographischen Isolation viel deutlicher von ländlichen Strukturen geprägt.

Im Rahmen meiner Feldforschungen Mitte der 90er Jahre wurden unter der damaligen Schulbevölkerung in Fragebögen zum Sprachgebrauch und zu Spracheinstellungen (Abdruck des Fragebogens in Riehl 2001: 310-311.) auch der Dialektgebrauch bzw. der Gebrauch umgangssprachlicher Varietäten des Deutschen abgefragt. ${ }^{3}$ Insgesamt wurden 220 Schülerinnen und Schüler in den Schulorten Eupen und St. Vith und 270 Schülerinnen und Schüler im Schulort Bozen im Alter von 14-18 Jahren befragt. Die Schulorte decken sich nicht unbedingt mit den Wohnorten der Schüler: Gerade im Schulort Bozen kamen auch viele Schüler aus dem dörflichen Umland. ${ }^{4}$ Abgefragt wurden die klassischen Domänen Familie, Freunde, öffentlicher Bereich (hier: Geschäft) und Institutionen (hier: Sprachgebrauch mit Lehrern cf. Riehl 1999).

In der Domäne Familie gaben in Eupen nur 3\% an, den Dialekt immer zu verwenden, 6\% oft, dagegen $31 \%$ nur manchmal und $60 \%$ nie. In St. Vith trugen dagegen $42 \%$ immer, $13 \%$ oft, $23 \%$ manchmal und $22 \%$ nie ein. Noch reduzierter ist das Bild in der Domäne Freunde. So verwenden laut Angaben meiner Informanten in Eupen 3\% immer, 6\% oft, 14\% manchmal und $72 \%$ nie in Gesprächen mit Freunden den Dialekt. In St. Vith dagegen gebrauchen die Informanten in dieser Domäne zu 28\% immer, 18\% oft, 27\% manchmal und 27\% nie den Dialekt. Es zeigt sich hier bereits die erwähnte Differenzierung an: Während im Großraum Eupen nahezu 2/3 der Schülerinnen und Schüler den Dialekt nicht sprechen, sind dies im St. Vither Raum nur 22\%. D. h. der Dialekt hat in diesem Gebiet, zumindest im informellen Bereich - besonders im Bereich der Familie -, noch einen hohen Stellenwert.

Für die Sprachverwendung im öffentlichen Bereich ist die Situation dagegen anders: Für den Bereich Geschäft gaben für Eupen $0 \%$ immer, 3\% oft, 15\% manchmal und $82 \%$ nie an, in St. Vith sehen die Angaben wie folgt aus: 4\% immer, 28\% oft, 30\% manchmal, 38\% nie. Am wenigsten wird erwartungsgemäß der Dialekt im institutionellen Bereich - hier mit Lehrern verwendet, in Eupen nur bei $6 \%$ der Informanten manchmal, bei $94 \%$ nie. In St. Vith gebrauchen immerhin 30\% der Schüler manchmal den Dialekt gegenüber den Lehrern, aber auch hier die überwiegende Mehrheit (70\%) nie (cf. Abb. 1 und 2). D. h. es wird erkennbar, dass der Dialekt im Wesentlichen auf die familiäre Sphäre und den Bereich Freunde beschränkt bleibt. Allerdings ist in diesem Zusammenhang zu bemerken, dass in Ostbelgien in bestimmten Bereichen die regionale Umgangssprache ("unser Deutsch", s. u. Kap. 4, Bsp. 3) den Dialekt ersetzt (s. u.).

\footnotetext{
3 Die Daten sind aufgrund der Minderheitensituation bis auf geringe Verschiebungen noch immer aktuell. Vor allem in Bezug auf die Relationen zwischen dem Varietätengebrauch in den verschiedenen Domänen und der Entwicklung in den beiden Minderheitengebieten gibt es keine Veränderungen.

4 Die Herkunftsorte der Schüler wurden aber ebenfalls mit erhoben und können Aufschluss über ein mögliches Stadt-Land-Gefälle geben. Da das für die hier untersuchte Fragestellung nur wenig relevant ist, wird im Folgenden nicht im Einzelnen darauf eingegangen.
} 


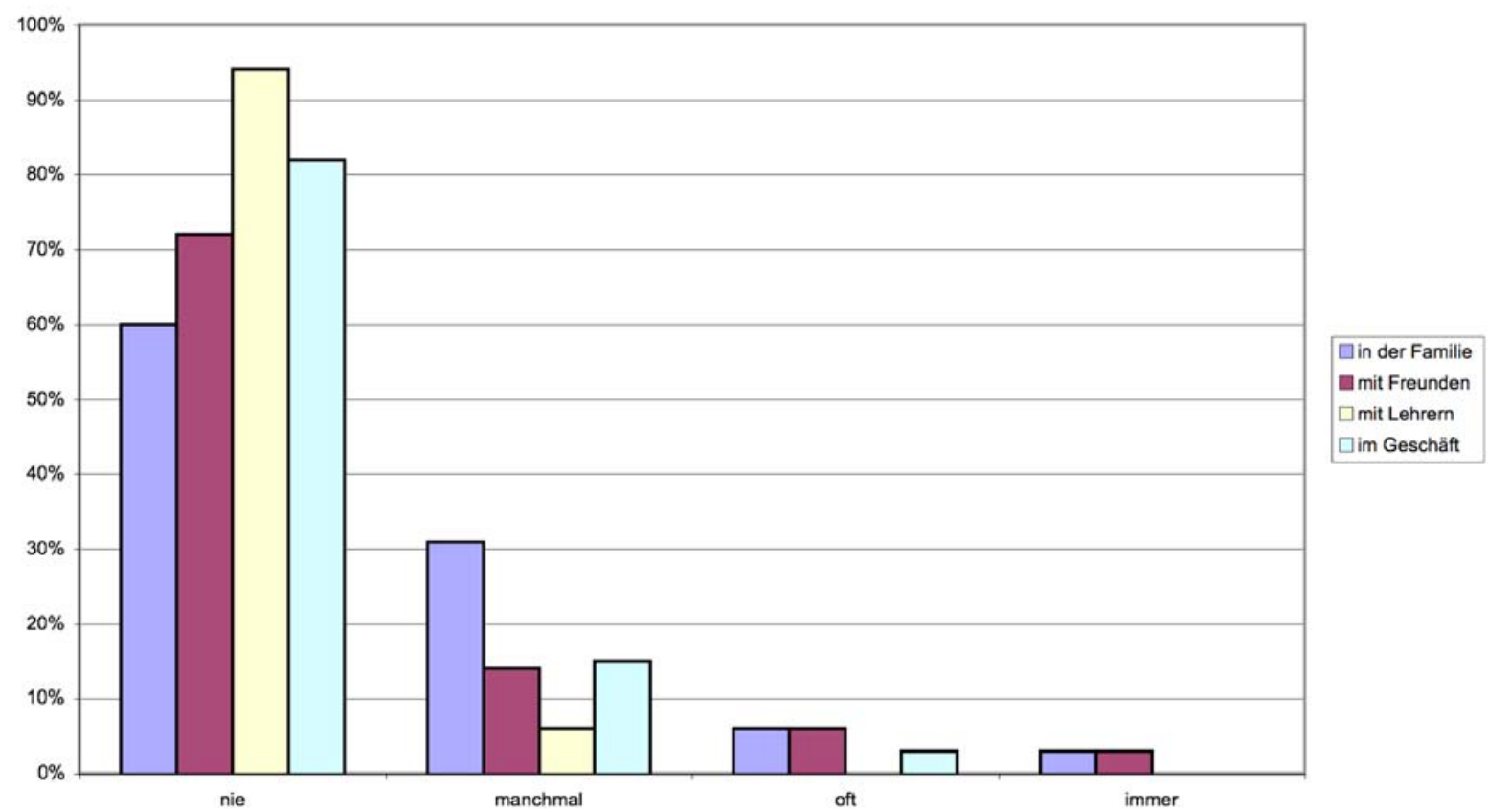

Abb. 1: Dialektgebrauch in Eupen

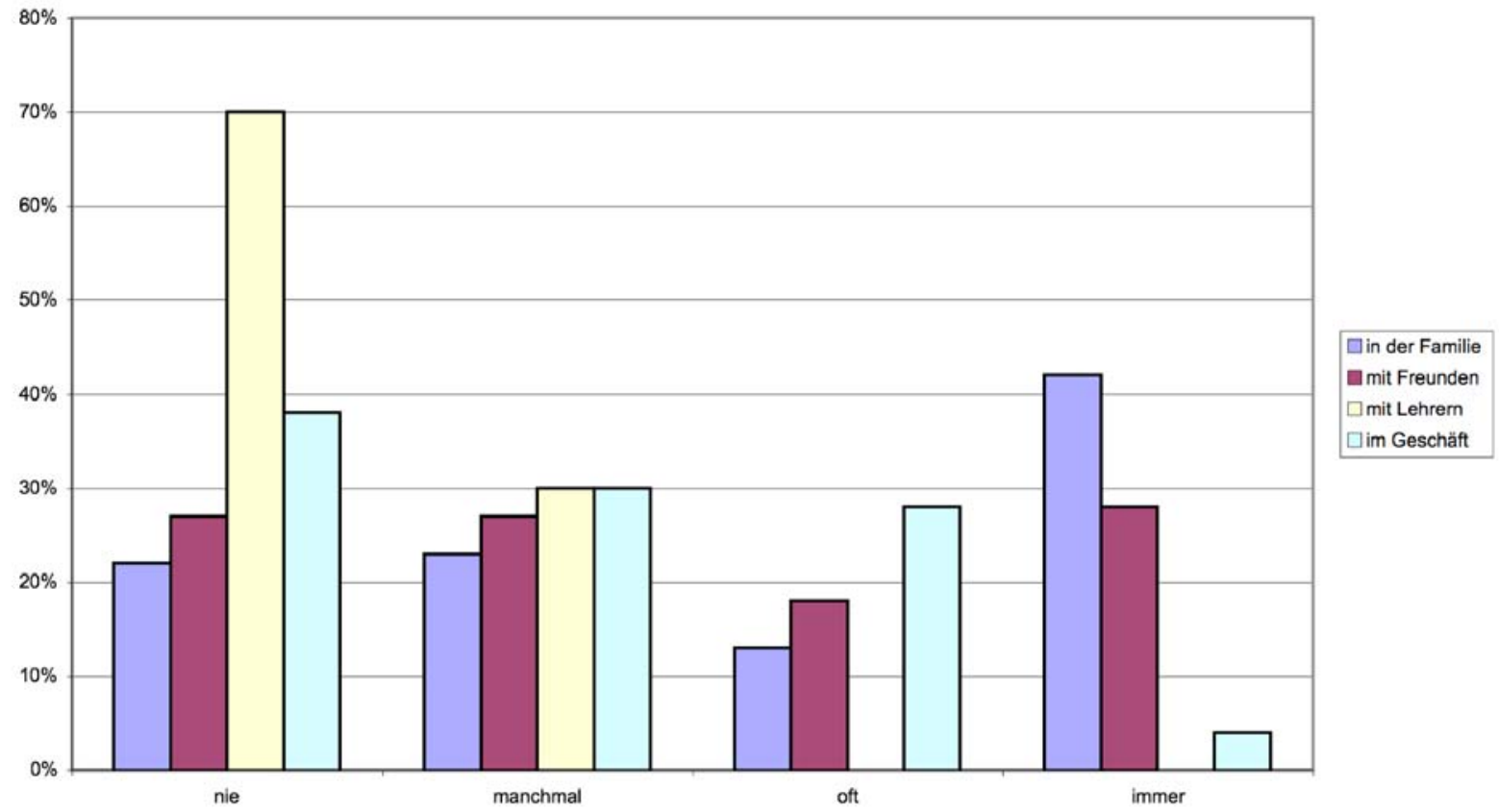

Abb. 2: Dialektgebrauch in St. Vith

Ganz anders präsentiert sich das Bild in Südtirol: Hier gebrauchen von den 270 befragten Schülerinnen und Schülern in der Domäne Familie zu $80 \%$ immer, zu $10 \%$ oft, zu $6 \%$ manchmal und nur zu 4\% nie den Dialekt (davon stammen 3\% aus rein italienischsprachigen Familien). Im Gegensatz zu den ostbelgischen Daten wird in den Gesprächen mit den Freunden sogar noch öfter der Dialekt verwendet als in der Familie, hier zu 82\% immer, 14\% oft, 4\% manchmal und 0\% nie. Daraus lässt sich also schließen, dass der Druck in Südtirol allgemein sehr groß ist, in möglichst vielen Domänen Dialekt zu verwenden. Das wird besonders an Beispielen deutlich, wo Schülerinnen und Schüler, die zuhause keinen Dialekt sprechen (weil sie beispielsweise Italienisch sprechen), mit den Schulkameraden trotzdem den 
Dialekt verwenden. D. h. alle Schüler beherrschen den Dialekt. ${ }^{5}$ Man muss von einer diglossischen Verteilung von Dialekt und Hochsprache ausgehen: Dialekt ist fast ausschließliches Kommunikationsmedium im Bereich der gesprochenen Sprache. Das Standarddeutsche (in seiner spezifisch regionalen Prägung) wird nur in formellen Sprechsituationen (auf Ämtern, im Unterricht, in der Kirche, bei offiziellen Ansprachen, vor Gericht u. ä.) und im Umgang mit Touristen oder den italienischsprachigen Mitbürgern verwendet. Teilweise erhält der Dialekt auch Einzug in die Medien und wird auch in bestimmten Gremien verwendet.

Die Bozener Informanten gaben an, in der öffentlichen Domäne (am Beispiel Geschäft) zu $28 \%$ immer, $40 \%$ oft, $29 \%$ manchmal und zu 3\% nie den Dialekt zu verwenden. Hier muss erläuternd angeführt werden, dass viele Geschäfte in Bozen italienischsprachig sind und dort automatisch die italienische Sprache verwendet wird. Dazu ist zu erwähnen, dass in der Kommunikation mit Angehörigen der italienischsprachigen Gruppe in allen Domänen viel häufiger die italienische Sprache gebraucht wird, da diese oft die deutsche Sprache weniger gut beherrschen oder aber den Dialekt nicht sprechen. Dies hängt offensichtlich mit einem psychologischen Problem zusammen: Für die deutschsprachigen Südtiroler bedeutet es eine größere Hemmschwelle, Standarddeutsch zu sprechen als Italienisch. Dies gilt besonders für informelle Situationen, die Domänen der Nähesprache sind (cf. auch Lanthaler 1990).

In der institutionellen Kommunikation (hier am Beispiel Lehrer) geben 0\% an, immer den Dialekt zu gebrauchen, $2 \%$ tun dies oft, $63 \%$ manchmal und 35\% nie (cf. Abb. 3). In diesem Fall macht sich der starke Normdruck der Schule bemerkbar: In der Minderheitensituation kommt aufgrund des geringeren Anteils deutschsprachiger Medien und des Kontakts mit Sprechern aus anderen deutschsprachigen Gebieten der Schule eine besonders wichtige Rolle in der Vermittlung des Standards zu (Riehl 1994). ${ }^{6}$

\footnotetext{
${ }^{5}$ Ein Beispiel: Ein 19jähriger Schüler italienischsprachiger Eltern, der in München aufwuchs, kam mit zwölf Jahren nach Bozen und wurde als Piefke beschimpft. Er hat sich daraufhin sofort den Dialekt angeeignet.

${ }^{6}$ Es fällt auf, dass Dialektsprecher, die auf ihren Basisdialekt beschränkt sind, große Schulschwierigkeiten haben (auch in der Zweitsprache). Dialektsprecher, die auch über standardnahe Register verfügen, haben kaum Probleme. D. h. hier ist - wie auch Rosenberg (1993) betont - nicht die dialektfreie Rede, sondern die Variationskompetenz von Bedeutung.
} 


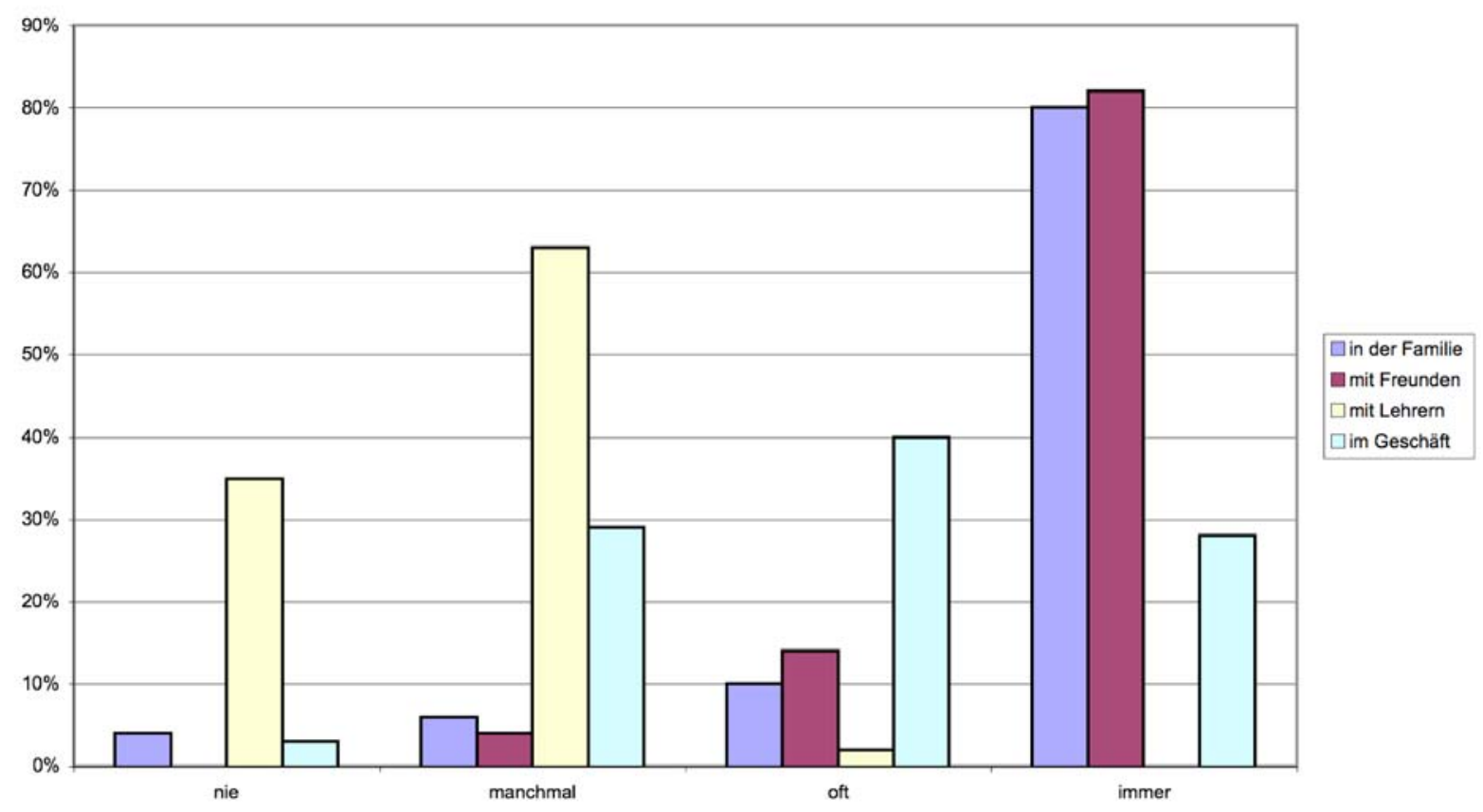

Abb. 3: Dialektgebrauch in Bozen

In Bezug auf Südtirol wurde immer die Problematik diskutiert, es gebe keine Konvergenzund Divergenzprozesse zwischen den beiden Varietäten (das sog. Bozener Deutsch ist wenig prestigereich), da auch die Tendenz bestehe, eher Italienisch statt Standarddeutsch zu verwenden (s. o.). Allerdings hat bereits Lanthaler (1997) darauf hingewiesen, dass Ausgleichserscheinungen festzustellen sind, die sich aufgrund der stärkeren Mobilität und der geänderten Lebensbedingungen ergeben. Es entsteht also eine Art Ausgleichsdialekt im syntaktischen und lexikalischen Bereich, der regionale Lautvarianten zulässt. Auf der anderen Seite ist eine Anpassung an Prestigevarietäten (prestigeträchtige Dialekte, städtische Umgangssprachen) zu bemerken. Die regionale Varietät 'Südtiroler Standarddeutsch' zeigt außer der regionalen Färbung im lautlichen Bereich typische Charakteristika bairischösterreichischer regionaler Standards (z. B. Entlehnung hochfrequenter dialektaler Formen), aber auch einige Übernahmen aus der Kontaktsprache (cf. Riehl 2000, zur Entstehung von Zwischenregistern Lanthaler 2001).

\section{$4 \quad$ Einstellung gegenüber Dialekt und anderen Varietäten}

Der Gebrauch von Dialekt und regionalen Varietäten in bestimmten Domänen ist vor allem von der Einstellung der Sprecher gegenüber diesen Varietäten abhängig. Es ist davon auszugehen, dass Dialekte und regionale Varietäten in Sprachminderheitsgebieten im Allgemeinen eine positive Bewertung von Seiten ihrer Sprecher erfahren (cf. dazu Riehl 2002). ${ }^{7}$ Doch ist in diesem Zusammenhang zu hinterfragen, inwieweit der Dialekt von den Sprachminderheiten als Identitätssymbol operationalisiert wird. Hier muss man zunächst auf die unterschiedliche Bedeutung der Sprachen und Varietäten für die Konstruktion von Identität hinweisen.

Die Identifikation mit einer bestimmten Ethnie ist bei Minderheiten, die einer anderssprachigen und anderskulturellen Nation angehören immer problematisch. ${ }^{8}$ Allerdings zeigt ein Vergleich von Aussagen ladinischsprachiger Südtiroler und deutschsprachiger

\footnotetext{
${ }^{7}$ Zur allgemein positiven Einstellung gegenüber Dialekt in Österreich und Südtirol cf. auch Steinegger (1998).

${ }^{8}$ Zur Problematik und Beziehung von Grenze, Sprache und Raum cf. Auer (2004).
} 
Südtiroler, dass gerade im letzteren Fall die Situation weit komplexer ist (cf. dazu Riehl 2002). Denn im Gegensatz zu einer nationslosen Minderheit sprechen diese eine Sprache, die in anderen Nationen Staatssprache ist. Aus diesem Grund operationalisieren die Südtiroler den Südtiroler Dialekt als zentrales Identifikationsmoment, wie sich in vielen Interviews herauskristallisierte. ${ }^{9}$ Sie konstruieren so eine eigene Identität als "Südtiroler": Südtiroler sind Angehörige einer Gruppe, die einen bestimmten Dialekt sprechen:

Bsp. (1)

\section{Sprecher A: 18 Jahre, deutschsprachig}

1 A: $\quad$ ich glaub, dass sich die (--) die deutschsprachigen Südtiroler jetzt eine eigene (-) 2 Iden-

2 tität irgendwie aufgebaut haben, indem sie ihren Dialekt noch sprechen, (-) sie identi-

3 fizieren sich da mit allen anderen, die diesen besonderen Dialekt dann sprechen,

4 (--) dies sind dann die Südtiroler. [...] und nicht (-) äh und nichts zu tun mit den Ös-

5 terreichern, (-) und Italiener sind Italiener, italienischsprechend. wir sind Deutsche, (---)

6 CR: $\quad \mathrm{mhm}, \mathrm{mhm}(--)$ glaub/, glaubt ihr

7 A: deutsch im Sinn von deutschsprachig.

Hier bringt es der Sprecher ganz klar zum Ausdruck: die Identität der Südtiroler manifestiert sich in ihrem Dialekt. Diejenigen, die diesen Dialekt sprechen (Z. 3f.) gehören zur 'In-group', zur Gruppe der Südtiroler. Diese grenzen sich nach zwei Seiten hin ab: einmal von Österreich, zu dem sie ursprünglich gehörten, und einmal von Italien. Dabei wird 'deutsch sein' mit 'deutschsprachig sein' (Z. 7) gleichgesetzt. Die kulturelle Identität wird unmittelbar mit der sprachlichen Identität verknüpft, "Deutsch" versteht man nicht im Sinne einer ethnischen Zuordnung, sondern rein auf sprachlicher Ebene.

Das Beispiel lässt damit die starke Betonung der regionalen Sprachvarietät erkennen, vorrangig vor der regionalen Kultur: Der Dialekt bekommt einen hohen Symbolwert. Ein Grund dafür ist, dass die Diskussion, die die Minderheiten führen, auf der Sprachgruppenzugehörigkeit basiert. Dadurch ist auch die Meinung sehr weit verbreitet, dass Kultur unmittelbar an Sprache gebunden sei. Ein Argument, das in diesem Zusammenhang ins Feld geführt wird, ist daher, ohne Sprache gehe auch die Kultur verloren, cf.:

Bsp. (2)

Sprecher A: 18 Jahre, deutschsprachig

1 A: wenn wir jetzt (--) alle Italienisch sprechen würden dort in der Schule, also nur Italie2 nisch hätten, (--) und kein Deutsch, oder höchstens als Fremdsprache so, nur italie3 nischsprachigen Unterricht, dann (--) würden wir auch die italienische (-) Mentalität $4 \quad$ annehmen, (-) und die eigene Kultur wird so langsam verloren gehen.

Hier bringt der Sprecher zum Ausdruck, dass ein Modell der Zweisprachigkeit, wie es in Südtirol praktiziert wird, die einzige Möglichkeit bietet, die eigene Kultur zu erhalten. Die Übernahme der italienischen Sprache würde bedeuten, dass damit die deutsche Kultur verloren gehen würde (Z. 4). Das ist eine Meinung, die keinen Einzelfall darstellt, sondern von vielen anderen Informanten, auch älteren, geteilt wird: Sprache und Kultur gehören unmittelbar zusammen, ohne Sprache kann die Kultur nicht erhalten werden. Selbst auf provokatives Fragen der Interviewerin hin, warum man beispielsweise nicht einfach die Sprache wechseln und doch die Kultur erhalten könne, gaben die Befragten stets zur Antwort, dass dies nicht möglich sei. Begründet wurde dies damit, dass Bräuche und andere kulturelle Charakteristika nicht ohne Sprache gedacht werden könnten. Interessant an dem letzten

\footnotetext{
${ }^{9} \mathrm{Zu}$ den Interviews cf. Riehl (1997) und (2002). Die Beispiele sind ebenfalls aus diesen Artikeln entnommen.
} 
Beispiel ist, dass der Sprecher die Übernahme einer anderen Sprache auch mit der Übernahme einer anderen Mentalität koppelt (Z. 3f.) und dass er der Meinung ist, dass die Übernahme dieser Mentalität auch den Verlust der Kultur zur Folge hätte.

In der ostbelgischen Sprachgemeinschaft wird dagegen die Identifikation mit der deutschen Sprache und Kultur vielmehr als Wahl angesehen. Die ostbelgischen Sprecher betonen ihre Besonderheit gerade dadurch, dass sie ein eigenes Deutsch sprechen, das sich auch durch die Sprachmischung mit dem Französischen vom Binnenstandard unterscheidet:

Bsp. (3)

$\begin{array}{ll}\text { A: } & \text { Schüler, } 17 \text { Jahre, St. Vith } \\ \text { B: } & \text { Schüler, } 17 \text { Jahre, Burg Reuland }\end{array}$

I: $\quad$ Habt ihr irgendwie das Gefühl, dass euer Deutsch, das ihr hier sprecht, irgendwie $2 \quad$ anders ist als das, was in Deutschland gesprochen wird?

A: $\quad \mathrm{Ja}$, ich glaub schon. (--) Nein wir sprechen eigentlich hier, eigentlich eher unser Deutsch. Das ist klar, dass es hier abhängig von Deutschland is, aber äh (-) ich mein, wie ich jetzt momentan spreche, das ist eben unser Deutsch. Ich kann auch (-) äh (-) versuchen, (-) äh (-) ein dialektfreies Deutsch zu sprechen. Das geht auch. Oder einen anderen deutschen Dialekt zu imitieren, aber äh + ich mein, das ist dann nicht mehr unser Deutsch hier. [...] Weil ich glaube, die meisten äh (-) könnten auch so wie in Köln oder so oder so ähnlich (-) sprechen, aber das wird bewusst nicht gemacht.

Unser Deutsch (Z. 3f.) meint hier die besondere regionale Varietät, die aber als 'Dialekt' bezeichnet wird. Dies wird deutlich durch die Abgrenzung von ein dialektfreies Deutsch sprechen (Z. 6) und einem anderen deutschen Dialekt zu imitieren (Z. 7). ${ }^{10}$ Die Sprecherin ist sogar der Meinung, dass die Leute anders sprechen könnten, aber dies bewusst nicht tun (Z. 9f.). Im weiteren Verlauf des Gespräches wird aber deutlich, dass die Sprecher glauben, durch die Übernahme von Spezifika des moselfränkischen Dialekts, der als 'Platt' bezeichnet wird, eine eigene Sprachvarietät zu kreieren.

Während im Raum St. Vith "unser Deutsch" eine Umgangssprache ist, die eher durch das Dialektsubstrat beeinflusst wird, ist es in Eupen die französische Kontaktsprache, die sich auf die Herausbildung eines eigenen Deutsch auswirkt:

Bsp. (4)

A: $\quad$ Schüler, Eupen, 17 Jahre

B: $\quad$ Schüler, Eupen, 17 Jahre

A: $\quad$ Die [= die Deutschen] gucken einen nur manchmal komisch an, je nachdem, was man sacht, weil es gibt ja in Belgien viel so belgische Ausdrücke, die stehen gar nich im Wahrig drin. Die stehen aber bei uns in 'n Heften und Büchern drin. Zum Beispiel bei uns in der Schule heißt es immer, wir machen 'ne Abfrage. Korrekt heißt es ja Befragung oder Klausur oder Arbeit oder Test, was weiß ich, aber Abfrage gibt es nich. [...] Was gibt's noch? Kompass. Das is Zirkel. [...] Oder was noch? Klavier. Kann 'ne Tastatur sein vom Computer [...]

B: Wir werden auch manchmal komisch angeguckt. Je nachdem, was man sacht. Wenn man das weiß natürlich, dann kann man sich danach richten.

\footnotetext{
${ }^{10}$ Diese Unsicherheit der Unterscheidung Dialekt - Umgangssprache findet man in der gesamten Sprachgruppe. Das hängt damit zusammen, dass der tatsächliche Dialekt als 'Platt' bezeichnet wird.
} 
Die Sprecher führen hier einige Eigenheiten des belgischen Deutsch auf, die sie selbst als solche definieren und die sie auch bewusst einsetzen können, um dieses typische Deutsch zu sprechen. Dabei handelt es sich jedoch nur um lexikalische Besonderheiten, Wörter, die nicht im Wahrig drin stehen (Z. 2f.). Interessant auch die Bemerkung von B: wenn man zur Ingroup gehören will, muss man auch deren Sprache benutzen - sonst wird man komisch angeguckt (Z. 8). Dieses 'Komisch-angeguckt-werden' dient als Indikator dafür, dass man etwas Eigenes in der Sprache benutzt, das dem Gegenüber nicht geläufig ist. Der Einsatz dieser Besonderheiten kann bewusst oder unbewusst erfolgen.

Auch in diesem Fall ist jedoch zwischen den beiden Gebieten Eupen und St. Vith zu unterscheiden. Während im nördlichen Kanton die Zweisprachigkeit Deutsch-Französisch eine wichtige Rolle spielt, ist im südlichen St. Vither Raum eine stärkere Dialektloyalität zu vermerken. Damit einher geht allerdings auch eine geringere Kompetenz in der Zweitsprache (cf. Riehl 2001: 283). Grundsätzlich lässt sich in der gesamten Sprachgemeinschaft auch eine sehr positive Einstellung gegenüber dem Französischen vermerken, das allgemein als städtisch und modern gilt. ${ }^{11}$ Das hat zur Folge, dass eine Mischvarietät, die auch Entlehnungen aus dem Französischen enthält, als regionale Varietät akzeptiert wird.

\section{$5 \quad$ Kontakteinflüsse des Dialekts}

Kontakteinflüsse aus dem Dialekt sind natürlich im Gesprochenen häufig zu bemerken. Sehr häufig ist der sog. Input-Switch (Moosmüller 1991: 38), d. h. innerhalb einer Äußerung wird an unterschiedlichen Stellen einmal die dialektale Form und einmal die hochsprachliche Variante verwendet:

$$
\begin{aligned}
& \text { In Oberinn da sein vielleicht -- sagn ma am Ritten -- da sind hundert Italiener } \\
& \text { verstreut auf sieben Fraktionen }
\end{aligned}
$$

Die Korrektur des Sprechers zeigt ganz deutlich, dass er versucht, den Input-Switch von Standard in Dialekt an akzentuierten Positionen zu vermeiden. An wenig betonten Stellen wird statt des standardsprachlichen sind immer die dialektale Form sein verwendet.

\subsection{Lexikalische Einflüsse}

Dialektwörter finden sich v. a. im Corpus aus Südtirol. Hier lässt sich eindeutig ein höherer Anteil bei Schülern aus sehr stark ländlich und dialektal geprägten Gegenden Südtirols feststellen. Da die Heimatorte der Schüler ebenfalls abgefragt wurden, kann dieser Aspekt mit berücksichtigt werden. Der Anteil der Dialekteinflüsse nimmt allerdings von Klassenstufe zu Klassenstufe ab, cf.: 12

(6) a) [...] diese Traube aufzuklauben [= aufheben]

b) der Traubentschaggl [= Traube]

c) [...] da der Aufprall doch etwas fest war. [= stark]

d) Links hebt die böse Königin [...] den vergifteten Apfel in einer Hand. [= hält]

e) den ober dem Kaffeehafen gelegenen Teil [= über der Kaffeekanne]

f) [...] bis es knackst [= bis es 'Klick' macht]

g) bis der fertige Kaffee in die Kanne geronnen ist [= gelaufen]

\footnotetext{
11 Teilweise hat das sogar zur Folge, dass Deutschsprachige in Kelmis und Eupen ihre Kinder auf französischsprachige Züge schicken (dazu und zur Zweitsprachkompetenz allgemein cf. Wilken 2005).

12 Die folgenden Beispiele sind Riehl (2001:258-260) entnommen.
} 
Interessanterweise werden meist Lexeme verwendet, die auch in der Standardsprache existieren, allerdings in einer anderen Bedeutung (cf. fest, heben, knacksen, rinnen). Dies zeigt wiederum das Bewusstsein der Schreiber für in der deutschen Standardsprache existente Wörter. Wortmarken, die in dieser Varietät nicht vorhanden sind, werden weitgehend vermieden. ${ }^{13}$

Dialekteinflüsse auf lexikalischer Ebene zeigen sich in geringerem Maße auch im belgischen Corpus:
a) [...] als er nun die halb zerpratschten Trauben musterte. [= zermantscht]
b) $\quad \operatorname{der} \boldsymbol{M o ̈ p}$ [= Köter, hier: ungehobelter Mensch]
c) die kleine Weinklombe [= Weintraube]
d) Schütten Sie 3 Kaffeelöffel bei. [= hineinschütten]
e) [...] und holt sie mit zu einem wichtigen Geschäftstermin. [= mitnehmen]

Auch hier werden in Beispiel d) und e) in der Standardsprache vorhandene Lexemstämme verwendet, allerdings mit einem untypischen Präfix kombiniert. In Beispiel e) wird das Verb in einem für die Standardsprache untypischen Kontext verwendet. Dieser Gebrauch von holen in der Bedeutung von 'nehmen' ist jedoch in der rheinischen Umgangssprache allgemein verbreitet.

\subsection{Morphologisch-syntaktische Einflüsse}

Nur wenige Schüler in der Probandengruppe zeigen Einflüsse des Dialekts oder der regionalen Umgangssprache im Bereich der Morphologie. Die Beispiele konzentrieren sich zum Großteil auf Schüler aus dem 10. und 11. Jahrgang und auf die Schreiber aus Südtirol. So finden sich noch vereinzelt im Bereich der Verben, deren Partizip II im Dialekt schwach und in der Standardsprache stark flektiert wird, analog gebildete schwache Präteritumsformen wie scheinte, bratete, hebte, ist [...] abgehaut. Häufig ist auch die Nullflexion im Akkusativ bei den schwachen Substantiven (zeigt einen Prinz). Diese findet sich jedoch auch bei einsprachigen deutschen Schülern aus Dialektgebieten und spiegelt eine allgemeine Entwicklungstendenz des Deutschen wider.

Schüler aus Südtirol zeigen teilweise Dialekteinfluss im Bereich der Kasusrektion (a) und der Genera (b) sowie im Gebrauch des Artikels bei artikellosen Substantiven (c). Diese Transfererscheinungen treten aber nur bei Sprechern aus ausgesprochenen Dialektgebieten und bei Schülern in berufsbildenden Schulen auf:
a) Um ihr herum befinden sich viele Zwergroboter.
b) im linken oberen Eck (das Eck = die Ecke)
c) das Latein

Im ostbelgischen Corpus macht sich die Tatsache bemerkbar, dass sowohl in den dort angesiedelten Dialekten als auch in der Umgangssprache häufig Dativ und Akkusativ vertauscht werden. Trotzdem sind Fälle wie die in Bsp. (9) aufgeführten in schriftlichen Texten ziemlich selten:
a) [...] kam ein Wolf an die Weintraubenplantage vorbei.
b) [...] spaziert vor die Trauben umher.
c) im Behälter zurücktun
d) Man drücke auf dem Knöpfchen.

\footnotetext{
13 Dies ist ein Hinweis auf das sog. language tagging (Green 1998): Bestimmte Lexeme werden mit entsprechenden Sprachmarken versehen und sind im mentalen Lexikon nebeneinander gespeichert. Daher werden sie nicht immer unterschieden.
} 
Varietätengebrauch und Varietätenkontakt in Südtirol und Ostbelgien

In den Texten der Schüler aus Ostbelgien finden sich daneben Verwechslungen der Richtungsadverbien, d. h. eine Vertauschung der Präfixe hin- und her-:

(10) a) Plötzlich fallen die Weintrauben vom Strauch [...] hinunter.

b) Dann schaut er herauf [= zum Baum].

c) Sie legen den Behälter jetzt wieder herein.

d) Ziehen Sie den Filterbehälter hinaus.

Zwei Beispiele finden sich mit der regionalen Variante drin, nicht zufällig von zwei sehr stark im Dialekt sozialisierten Schülern:

(11) a) Setzen sie die gewünschte Kaffeemenge darin.

b) [...] und tun ihn mit der spitzen Seite nach unten drin.

In einigen Fällen wird in beiden Regionen das Relativpronomen wie in Umgangssprache/ Dialekt durch das Interrogativpronomen wo oder auch was ersetzt:

(12) a) eine Weinrebe, wo reife Trauben hingen. (Südtirol)

b) Wolkenkratzer, wo die sieben Roboter drin wohnen. (Belgien)

c) das Schloß, was sich auf dem Kopf des Schneewittchens befindet. (Belgien)

Wie die Beispiele zeigen, treten diese Fälle in beiden Minderheitengruppen auf, sind aber in der geschriebenen Sprache insgesamt als marginal einzustufen.

In diesem Zusammenhang ist jedoch zu diskutieren, inwieweit man hier von der Herausbildung eines regionalen Standards sprechen muss: Es werden sowohl im Südtiroler Deutsch als auch im ostbelgischen Deutsch neben Übernahmen aus der Umgebungssprache (Italienisch oder Französisch) Besonderheiten aus den zugrunde liegenden Dialekten in die Standardsprache integriert, die damit zur Herausbildung einer eigenen regionalen Standardvarietät beitragen könnten (cf. Riehl 1994, 2000) (zum Corpus Südtiroler Deutsch cf. Anstein in diesem Band).

\section{Zusammenfassung und Konsequenzen für Deutsch als Zweitsprache}

Südtiroler Sprecher operationalisieren ihren Dialekt als Symbol der eigenen Identität. Dies hat zur Konsequenz, dass der Dialekt das alleinige Kommunikationsmedium in gesprochener Sprache darstelltund typisch schriftsprachliche Register mit einer gewissen Verzögerung im Vergleich zu einsprachigen deutschsprachigen Gemeinschaften erworben werden. Damit sind die Kontakteinflüsse aus dem Dialekt zu erklären. Die Zweitsprache dagegen hat in Südtirol v. a. Einfluss bei primär Zweisprachigen oder bei sekundär Zweisprachigen, die in überwiegend italienischsprachigem Milieu aufgewachsen sind und sehr frühen und intensiven Kontakt zur italienischen Sprache hatten. (cf. Riehl 2001).

Ostbelgische Sprecher betonen ihre Besonderheit eher dadurch, dass sie die beiden Sprachen mischen. Hier ist jedoch zwischen den beiden Gebieten Eupen und St. Vith zu unterscheiden. Während im nördlichen Kanton die Zweisprachigkeit Deutsch-Französisch eine wichtige Rolle spielt, ist im südlichen St. Vither Raum, der auch viel stärker durch seine ländliche Struktur geprägt ist, eine stärkere Dialektloyalität zu vermerken. Diese geht gleichzeitig einher mit einer schwächeren Kompetenz in der Zweitsprache, was durch geringeren direkten Kontakt zu Französischsprachigen bedingt ist.

Aus diesen Beobachtungen ergeben sich auch einige wichtige Konsequenzen für den DaFUnterricht in zweisprachigen Gebieten: Wenn der Dialekt fast alleiniges Kommunikationsmedium in der gesprochenen Domäne ist, ist es wichtig, dass dieser auch im Zweitsprachunterricht vermittelt wird, zumindest eine passive Kompetenz (cf. dazu etwa den Beitrag von Putz in diesem Band). Dies ist vor allem auch deshalb von Bedeutung, da in gewissen Bereichen - wie die Ausführungen in Kapitel 5 zeigen - auch Kontakteinflüsse des 
Dialekts in schriftlichen Äußerungen feststellbar sind. Neben Einflüssen aus der Kontaktsprache können diese auch zur Ausformung eines eigenen regionalen Standards führen.

Eine weitere sehr wichtige Komponente muss ebenfalls mit vermittelt werden: Wie aus den in Kapitel 5 dargestellten Daten erkennbar wird, ist der Dialekt gerade für viele Sprachminderheiten ein Symbol der eigenen Identität. Nur über den Dialekt können sie eine eigene ethnische Identität aufbauen. Diese Notwendigkeit muss Lernern des Deutschen als Zweitsprache in diesen Gebieten deutlich gemacht werden, um ein entsprechendes Verständnis zu erzeugen. ${ }^{14}$ Dies ist besonders deshalb von Bedeutung, da Dialekte in den jeweiligen Kontaktgesellschaften weit negativer bewertet werden (etwa als 'bäuerlich' oder 'retro'). In Gebieten in denen der Dialekteinfluss weniger stark ist, kommt es dagegen darauf an, auf die Besonderheiten des regionalen Standards und der Umgangssprache aufmerksam zu machen.

\section{Transkriptionskonventionen}

$[\ldots]$

$(-)$

$(--)$

$(--)$

,

$\dot{?}$

$?$
Auslassung von Text

Pause bis 1 Sekunde

Pause von 2 Sekunden

Pause zwischen 3 und 5 Sekunden

steigende Intonation

fallende Intonation

Frageintonation

\section{Literaturangaben}

Auer, Peter (2004): "Sprache, Grenze, Raum". Zeitschrift für Sprachwissenschaft 23: 149179.

Egger, Kurt/Heller, Karin (1997): "Deutsch - Italienisch". In: Goebl, Hans et al. (eds.): Kontaktlinguistik. Ein internationales Handbuch zeitgenössischer Forschung. 2. Halbbd. Berlin/New York: 1350-1357.

Eichinger, Ludwig M. (1996): "Südtirol". In: Hinderling, Robert/Eichinger, Ludwig M. (eds.): Handbuch der mitteleuropäischen Sprachminderheiten. Tübingen: 199-262.

Green, David W. (1998): "Mental control of the bilingual lexico-semantic system." Bilingualism: Language and Cognition 1: 67-81.

Hinderdael, Michael/Nelde Peter H. (1996): "Deutschbelgien". In: Hinderling, Robert/ Eichinger, Ludwig M. (eds.): Handbuch der mitteleuropäischen Sprachminderheiten. Tübingen: 479-495.

Kern, Rudolf (1997): "Französisch-Deutsch in Belgien". In: Goebl, Hans et al. (eds.): Kontaktlinguistik. Ein internationales Handbuch zeitgenössischer Forschung. 2. Halbbd., Berlin/New York: 1130-1136.

Lanthaler, Franz (1990): "Dialekt und Zweisprachigkeit in Südtirol". In: Lanthaler, Franz (ed.): Mehr als eine Sprache. Zu einer Sprachstrategie in Südtirol. Meran: 57-81.

Lanthaler, Franz (1997): "Varietäten des Deutschen in Südtirol". In: Stickel, Gerhard (ed.): Varietäten des Deutschen: Regional- und Umgangssprachen. Berlin/New York: 364-383.

\footnotetext{
${ }^{14}$ Die fast ausschließliche Verwendung des Dialekts im gesprochenen Medium wird von der italienischen Sprachgruppe immer wieder beklagt (cf. Strobel 2002). Mit Projekten wie "Hoi Hanni" wurde bereits eine Initiative zur Verbreitung von zumindest passiven Kenntnissen im Dialekt gestartet. Dazu ist es aber auch notwendig, die eigentlichen Verstehensschwierigkeiten bei italienischen Muttersprachlern zu erheben (cf. dazu das Projekt von Putz in diesem Band).
} 
Lanthaler, Franz (2001): "Zwischenregister der deutschen Sprache in Südtirol". In: Egger/Lanthaler (eds.): Die deutsche Sprache in Südtirol. Einheitssprache und regionale Vielfalt. Wien/Bozen: 137-152.

Moosmüller, Sylvia (1991): Hochsprache und Dialekt in Österreich. Soziophonologische Untersuchungen zu ihrer Abgrenzung in Wien, Graz, Salzburg und Innsbruck. Wien/Köln/ Weimar.

Riehl, Claudia M. (1994): "Das Problem von 'Standard' und 'Norm' am Beispiel der deutschsprachigen Minderheit in Südtirol". In: Helfrich, Uta/Riehl, Claudia M. (eds.): Mehrsprachigkeit in Europa - Hindernis oder Chance? Wilhelmsfeld: 149-164.

Riehl, Claudia M. (1997): "Identité et conscience linguistique chez les minorités germanophones du Tyrol du Sud et de l'Est de la Belgique". In: Schneider-Mizony, Odile/ Gamer, Beate (eds.): Annales de l'Université de Savoie 22. Langue et identité. Savoie: 7997.

Riehl, Claudia M. (1999): "Zwischen Dialekt und Zweitsprache. Deutschsprachige Minderheiten und ihr Weg zum Standard". In: Stehl, Thomas (ed.): Dialekt, Dialektgenerationen, Sprachwandel. Tübingen: 133-149.

Riehl, Claudia M. (2000): "Deutsch in Südtirol". In: Wirrer, Jan (ed.): Minderheiten- und Regionalsprachen in Europa. Opladen: 235-246.

Riehl, Claudia M. (2001): Schreiben, Text und Mehrsprachigkeit. Zur Textproduktion in mehrsprachigen Gesellschaften am Beispiel der deutschsprachigen Minderheiten in Südtirol und Ostbelgien. Tübingen.

Riehl, Claudia M. (2002): "Italianità als Problem. Minderheiten und nationale Identität". In: Grimm, Reinhold R. et al. (eds.): Italianità. Ein literarisches, sprachliches und kulturelles Identitätsmuster. Tübingen: 115-131.

Rosenberg, Peter (1993): "Dialekt und Schule. Bilanz und Aufgaben eines Forschungsgebiets". In: Klotz, Peter/Sieber, Peter (eds.): Vielerlei Deutsch. Umgang mit Sprachvarietäten in der Schule. Stuttgart etc.: 12-58.

Steinegger, Guido (1998): Sprachgebrauch und Sprachbeurteilung in Österreich und Südtirol. Ergebnisse einer Umfrage. Frankfurt etc.

Strobel, Thomas (2002): Dauerhafte und aktuelle Problemkomplexe in Südtirol/Alto Adige. Kulturraumspezifisches Studienprojekt: Universität Passau.

Wilken, Marianne (2005): Verschiedene Wege zur Zweisprachigkeit. Empirische Untersuchung zur Zweisprachigkeit am Beispiel von Kindern in der Deutschsprachigen Gemeinschaft $(D G)$ in Ostbelgien. Dissertation, Philosophische Fakultät der RWTH Aachen. 